\title{
Reduced nitrogen in ecology and the environment
}

\author{
Special issue of the ESF-FWF Conference in \\ partnership with LFUI, October 2006 \\ J.W. Erisman \\ M.A. Sutton
}

Published in Environmental Pollution 154 (2008) 357 - 358 


\section{Reduced nitrogen in ecology and the environment: Special issue of the ESF-FWF Conference in partnership with LFUI, October 2006}

Nitrogen, contained in amino acids, proteins, and DNA, is essential for life. Much nitrogen is present in nature, almost all is in an unreactive form (gaseous nitrogen, $\mathrm{N}_{2}$ ) that is not usable by most organisms. The natural supply of reactive nitrogen in the environment through biological nitrogen fixation, biomass burning, volcanic activity and lightning is not sufficient to sustain the current abundance of human life. Thus humans learned in the early 20th century how to convert gaseous $\mathrm{N}_{2}$ into ammonia as a basis for fertilizers that could sustain food production. We industrially create $115 \mathrm{MT}$ of ammonia annually to sustain the relevant amino acids and other reduced nitrogen compounds in food. It is estimated that about $40 \%$ of the world's population depends on this production and use.

There are two major problems with nitrogen: some regions of the world do not have enough reactive nitrogen to sustain human life, resulting in hunger and malnutrition, while other regions have too much nitrogen (due mainly to the burning of fossil fuel and to the inefficient incorporation of nitrogen into food products) resulting in a large number of major human health and ecological effects. The rate of change of the problem is enormous and probably greater than that for other ecological problems. We foresee a steady increase in these problems as the expected increase in population and its wealth will strongly affect the primary drivers (food and energy) of the nitrogen issue. A prerequisite to reducing these problems is the development of a sound scientific basis on which to begin to discuss policy options. This Special issue provides an overview of important scientific challenges related to reduced nitrogen. It is based on papers presented at the ESF-FWF Conference in partnership with LFUI and Cost Action 729 on Reduced Nitrogen in Ecology and the Environment, organized in the Universitätszentrum Obergurgl, Austria on 14-18 October 2006 (www.esf.org/conferences/lc06203).

The scientific basis for reduced nitrogen in ecology and the environment needs to be strengthened. This Conference was the first major step to develop this basis. Reduced nitrogen is the least known part of the nitrogen cycle. Reduced nitrogen, such as ammonia, ammonium and amines are essential in food production, in ecology and also in the environment. So far, reduced nitrogen has not been regarded as a serious issue for the environment and only relevant on the local scale because of the short lifetime in the environment. The production and use of fertilizer, industrial activities and traffic (three-way catalyst) lead to emissions of reduced nitrogen to the environment. The biggest source, however, is related to animal excretion in extensive livestock breeding. Emissions of ammonia to the atmosphere can contribute to particulate matter formation affecting human health, decreasing biodiversity, contributing to eutrophication and acidification of aquatic and terrestrial ecosystems after deposition and to nitrous oxide formation, contributing to the greenhouse effect. The increased application rates of reduced nitrogen can cascade through the environment and contribute to different effects until in the cascade the reactive nitrogen is stored or converted back into $\mathrm{N}_{2}$. The scale of these environmental effects has been extended from a few hotspot regions such as Denmark, the Netherlands, parts of France, the Po Valley, to the whole of Europe. There are clear signals that the effects of reduced forms of nitrogen in the environment can be different from the oxidized forms especially in relation to ecosystem effects and biodiversity. The most important findings of the Conference are summarised in this Special issue and in an overview paper by Erisman et al. (2007) in the 150 Volume of Environmental Pollution.

\section{Gaps in our knowledge}

The ESF Conference showed clearly that there is a need for a new thorough updating of the Nitrogen cycle of at least the anthropogenic part, improving data on fluxes, reservoirs and also on uncertainties. This should be done for regions and globally. It is proven that reduced nitrogen is an expanding environmental problem and increasing because of the predicted growth in fertilizer use for food production and fossil fuel and biomass use for energy. The relationship between fertilizer use, biofuels and the environment is a topic that needs to be addressed in the coming future. Another issue is quantification of the nitrogen cascade where one molecule of reactive nitrogen can contribute to different environmental effects in time and possibly space. The question is if the cascade really happens, what the storage capacity is of the different systems and how much $\mathrm{N}$ is converted back by de-nitrification. The eutrophying effect of nitrogen deposition has also caused significant 
loss of biodiversity in some sensitive, nutrient limited ecosystems and this needs further quantification. Biodiversity and other ecosystem effects might be more sensitive to reduced forms of nitrogen than to oxidized forms.

Nitrogen plays an important role in carbon sequestration in aquatic and terrestrial ecosystems, stimulating growth and thereby $\mathrm{CO}_{2}$ uptake. However, this relationship is not linear and varies in space, time and type of system, with overall effects on soil carbon rather uncertain. This needs to be further explored. Next to this, the additional nitrous oxide emissions resulting from increasing fertilizer use for biofuel production remain a key trade-off that must be considered. There is large uncertainty on the direct and indirect emissions of $\mathrm{N}_{2} \mathrm{O}$ and thus doubts remain concerning the carbon neutrality of biofuels.

In agricultural systems the nitrogen balance can still not be quantified. There is a big gap in estimated nitrogen balances at different scales. Much farm-level research has shown that the gap between input and output of $\mathrm{N}$ can be up to $40 \%$. It is suspected that de-nitrification is the most important process that can explain the gap, even though organic nitrogen, which is often not fully included in $\mathrm{N}$ balances, might play a role. Nitrogen efficiency in crop and/or meat production never reaches $100 \%$. We always have to take into account losses to the environment. Depending on the system, the maximum nitrogen efficiency feasible is $40-50 \%$. There is a need of a comprehensive assessment of reduced nitrogen Best Management Practices and how technology can be brought to lead to further reductions of reduced gases.

Inventories of agricultural emissions are becoming available for different areas of Europe and North America, but there is a lack in other areas. The spatial scale needs to be refined in order to meet the requirements of dispersion models to assess the transport and deposition. Furthermore, the temporal variation in emission factors can be very large both from day to day as well as during the seasons. When using emission inventories the temporal variation needs to be addressed separately. Similarly, there remain significant industrial and energy and transport sources of reduced nitrogen and $\mathrm{N}_{2} \mathrm{O}$, as well as the well-known sources of $\mathrm{NO}_{x}$. For example, energy production and vehicle technologies to reduce $\mathrm{NO}_{x}$ emissions are leading to increases in both $\mathrm{N}_{2} \mathrm{O}$ and $\mathrm{NH}_{3}$ emissions.

We think that for a sustainable future with enough food and energy for the people it is of great importance to increase the attention for the ecological and environmental aspects of reduced nitrogen.

\section{Acknowledgement}

This special issue is the result of the ESF-FWF Conference on Reduced Nitrogen in Ecology and the Environment, organized in the Universitätszentrum Obergurgl, Austria on October 14-18, 2006 (http://www.esf.org/conferences/ lc06203). This Conference was organized by the European Science Foundation (ESF) in partnership with the Fonds zur Förderung der wissenschaftlichen Forschung in Österreich (FWF) and the Leopold-Franzens-Universität Innsbruck (LFUI). We also acknowledge the support of the COST Action 729, and the ESF program Nitrogen in Europe (NinE).

\section{Reference}

Erisman, J.W., Bleeker, A., Galloway, J., Sutton, M.S., 2007. Reduced nitrogen in ecology and the environment. Environmental Pollution 150 (1), $140-149$.

Jan Willem Erisman* Energy Research Centre of the Netherlands (ECN), P.O. Box 1, 1755 ZG Petten, The Netherlands

*Corresponding author. E-mail address: erisman@ecn.nl

Mark A. Sutton

Centre for Ecology and Hydrology, Edinburgh Research Station, Bush Estate, Penicuik, Midlothian, Scotland EH26 OQB, UK 